\title{
microRNA-21 overexpression contributes to cell proliferation by targeting PTEN in endometrioid endometrial cancer
}

\author{
XIAOYAN QIN ${ }^{*}$, LEI YAN ${ }^{*}$, XINGBO ZHAO, CHUNYAN LI and YIBING FU \\ Department of Obstetrics and Gynaecology, Provincial Hospital Affiliated to Shandong University, Jinan 250021, P.R. China
}

Received March 30, 2012; Accepted June 28, 2012

DOI: 10.3892/ol.2012.896

\begin{abstract}
The aim of this study was to investigate the role of microRNA-21 (miR-21) in the regulation of phosphatase and tensin homolog deleted from chromosome-10 (PTEN) expression and proliferation of endometrioid endometrial cancer (EEC) cells. We performed a qRT-PCR assay with miR-21 and PTEN in 16 paired EEC tumor tissues and adjacent non-tumor endometrium. To investigate the regulation of PTEN by miR-21, we designed gain- and loss-of-function of miR-21 experiments in the KLE cell line by transfection with a synthetic miR-21 mimic and inhibitor. To validate the putative binding site of miR-21 in the $3^{\prime}$ untranslated region (3'-UTR) of PTEN messenger RNA (mRNA), a dual-luciferase reporter assay was carried out. To evaluate the potential effect of miR-21 on EEC proliferation, we performed both overexpression experiments, using an miR-21 mimic, and inhibition assays, using an miR-21 inhibitor. miR-21 was overexpressed in EEC and was inversely correlated with PTEN protein expression $(\mathrm{P}<0.001)$. miR-21 regulated PTEN protein expression and cell proliferation in the KLE cell line and the direct binding of miR-21 to the PTEN 3'-UTR was confirmed using a dual-luciferase reporter assay. The upregulation of miR-21 led to a significant decrease in the PTEN protein expression level $(\mathrm{P}=0.007)$. The downregulation of miR-21 led to a significant increase in PTEN protein $(\mathrm{P}=0.002)$. The expression of luciferase in the wt-PTEN-3'-UTR-pGL3 group was downregulated in the presence of the miR-21 mimic $(\mathrm{P}=0.001)$. miR-21 was overexpressed in EEC. In conclusion, we demonstrated that the expression of PTEN protein, but not mRNA, was negatively directly regulated by miR-21 in the KLE cell line. The
\end{abstract}

Correspondence to: Dr Yibing Fu, Department of Obstetrics and Gynaecology, Provincial Hospital Affiliated to Shandong University, No. 324 Jing Wu Road, Shandong, Jinan 250021, P.R. China

E-mail: fu_yibing@yahoo.cn

*Contributed equally

Key words: miR-21, PTEN, endometrioid endometrial cancer, proliferation overexpression of miR-21 modulated EEC cell proliferation through the downregulation of PTEN.

\section{Introduction}

Endometrial cancer is the most common malignancy of the female genital tract in Western countries, with an estimated 43,470 cases and 7,950 mortalities due to the disease expected in the United States in 2010 (1). Of these cancers, 80-90\% are endometrioid endometrial cancer (EEC). Phosphatase and tensin homolog deleted from chromosome-10 (PTEN) is a tumor-suppressor gene. Functional inactivation of PTEN has been associated with EEC initiation and progression. A previous study reported a $34-55 \%$ somatic mutation frequency of the PTEN gene with a 50-83\% frequency of loss or decrease of the PTEN protein (2) in EEC. Thus, PTEN inactivation in EEC and in several other tumor types cannot be explained solely by observed mutations (3). However, the mechanism involved in the inactivation of PTEN is largely unknown.

microRNAs (miRNAs), whose final product is a 19-22 nt functional RNA molecule, are a class of short non-coding RNAs and play important regulatory roles by sequence-specific base pairing on the $3^{\prime}$ untranslated region (3'-UTR) of target messenger RNAs (mRNAs), promoting mRNA degradation or inhibiting translation (4). In addition, a widespread dysregulation of miRNAs is commonly observed in human cancer and promotes cellular transformation and tumorigenesis. miRNAs may function as oncogenes or tumor suppressors in tumor development (5). Specifically, miR-21 has been found to be overexpressed in numerous different solid tumors. Altered miR-21 has been found to be associated with the proliferation, invasion and apoptosis of malignant cells by targeting and downregulating a number of tumor suppressors, including PTEN (6,7), programmed cell death 4 (PDCD4) (8-11), bcl-2 and tropomyosin 1 (TPM1) (12-13), bone morphogenetic protein receptor II (BMPRII) (14) and sprouty 2 (SPRY2) (15). These findings imply that miR-21 plays a fundamental role in malignant behavior and transformation. A recent study reported that upregulated miR-21 was correlated with EEC (16). However, the authors did not show evidence of the role that miR-21 may play in EEC and whether or not this occurs through the PTEN pathway. Therefore, we designed this study with the aim of investigating the oncogenic role of miR-21 in the process of EEC and in the possible regulation of PTEN expression. 


\section{Materials and methods}

Tissues and cell line. Paired fresh tumor tissue and matched adjacent non-tumor tissues ( $>2 \mathrm{~cm}$ from the tumor) were obtained from 16 untreated EEC patients (aged from 33 to 72 years, with a mean age of $53.63 \pm 9.79$ ) who underwent surgery in our hospital. The samples were snap-frozen in liquid nitrogen, and appropriate informed consent and approval from the Human Research Ethics Committee were obtained. The risk factors of these patients included obesity and anovulation. The tissues were carefully dissected by the pathologist.

The PTEN-positive KLE cell line was obtained from the Type Culture Collection of the Chinese Academy of Sciences (Beijing, China) and was cultured in Dulbecco's Modified Eagle's Medium (DMEM; Hyclone, South Logan, UT, USA) supplemented with $10 \%$ fetal bovine serum (Hyclone) and $100 \mathrm{U} / \mathrm{ml}$ penicillin/streptomycin. The cultures were maintained at $37^{\circ} \mathrm{C}$ in a $5 \% \mathrm{CO}_{2}$ humidified chamber.

Transfection with miR-21 mimic and inhibitor. Mature hsa-miR-21 mimic, hsa-miR-21 inhibitor and their respective miRNA scrambled control (SC; Table I) were designed and chemically synthesized by GenePharma (Shanghai, China). $5 \times 10^{5} \mathrm{KLE}$ cells were seeded in 6-well plates (Corning, Lowell, MA, USA) and cultured in antibiotic-free medium for $48 \mathrm{~h}$ to achieve $>70 \%$ confluence on the day of transfection. The miR-21 mimic, inhibitor or their respective SC (40 nM) was transfected into cells using Lipofectamine ${ }^{\mathrm{TM}} 2000$ Reagent (Invitrogen, Carlsbad, CA, USA) in serum-free conditions for $6 \mathrm{~h}$ before changing to complete medium. The efficiency of transfection was determined using a Leica DMIRE2 microscope system (Leica Microsystemes, Montreal, QC, Canada). RNA and protein were collected 48 and $72 \mathrm{~h}$ after transfection, respectively.

RNA extraction and qRT-PCR assay. To test the expression of miRNA-21 and PTEN mRNA in EEC tissues and the KLE cell line, qRT-PCR was carried out using the ABI 7500 Real Time PCR system (Applied Biosystems, Foster City, CA, USA). For detecting mature miR-21, reverse transcription and quantitative PCR were performed using the Hairpin-it ${ }^{\mathrm{TM}}$ miRNAs qPCR Quantitation kit (GenePharma). U6 small nuclear RNA (snRNA) expression was assayed for normalization. The thermal cycling conditions for miR-21 and U6 snRNA consisted of $95^{\circ} \mathrm{C}$ for $3 \mathrm{~min}, 40$ cycles of $95^{\circ} \mathrm{C}$ for $12 \mathrm{sec}$ and $62^{\circ} \mathrm{C}$ for $1 \mathrm{~min}$. To detect PTEN mRNA, the RevertAid ${ }^{\mathrm{TM}}$ First Strand cDNA Synthesis kit (MBI Fermentas, Burlington, ON, Canada) was used and SYBR ${ }^{\circledR}$ Premix Ex Taq ${ }^{\mathrm{TM}}$ II (Takara, Dalian, China) was used (primers shown in Table II). The conditions for the PTEN and GAPDH reaction mixtures were $95^{\circ} \mathrm{C}$ for $2 \mathrm{~min}, 40$ cycles of $95^{\circ} \mathrm{C}$ for $15 \mathrm{sec}$ and $60^{\circ} \mathrm{C}$ for $1 \mathrm{~min}$ and finally $95^{\circ} \mathrm{C}$ for $15 \mathrm{sec}, 60^{\circ} \mathrm{C}$ for $1 \mathrm{~min}, 95^{\circ} \mathrm{C}$ for $15 \mathrm{sec}$ and $60^{\circ} \mathrm{C}$ for $15 \mathrm{sec}$. All experiments were performed in triplicate. Analysis of relative miR-21 and PTEN mRNA expression was performed using the comparative CT method with U6 and GAPDH snRNA as endogenous controls, respectively.

Western blot analysis. Protein was extracted from the tissue samples and cells $72 \mathrm{~h}$ after transfection using a protein extraction reagent (Beyotime, Shanghai, China) and protein
Table I. Sequences of miR-21 mimic, inhibitor and scrambled controls.

Name Sequence (5'-3')

hsa-miR-21 mimic

Sense

Antisense

UAGCUUAUCAGACUGAUGUUGA

SC for miR-21 mimic

Sense AACAUCAGUCUGAUAAGCUAUU

Antisense

UUCUCCGAACGUGUCACGUTT

hsa-miR-21 inhibitor ACGUGACACGUUCGGAGAATT

SC for miR-21 inhibitor CAGUACUUUUGUGUAGUACAA

SC, scrambled control.

Table II. Primers used in the qRT-PCR assay.

\begin{tabular}{lc}
\hline Primer name & \multicolumn{1}{c}{ Sequence (5'-3') } \\
\hline PTEN (accession no. NM_000314) \\
Forward & GCGTGCAGATAATGACAAGG \\
Reverse & GGATTTGACGGCTCCTCTAC \\
GAPDH (accession no. NM_002046) \\
Forward \\
Reverse \\
CAAGGTCATCCATGACAACTTTG
\end{tabular}

concentration was measured using the BCA Protein Assay kit (Beyotime). The proteins $(60 \mu \mathrm{g})$ were separated on $10 \%$ SDS-polyacrylamide gels and transferred to nitrocellulose filter membranes (Hybond, Escondido, CA, USA). The membrane was blocked with Tris-buffered saline Tween-20 (TBST) containing 5\% skimmed milk powder for $1 \mathrm{~h}$ at room temperature, followed by incubation in TBST containing 5\% BSA (Sigma, St. Louis, MO, USA) and primary antibodies overnight at $4^{\circ} \mathrm{C}$. Primary antibodies were detected using a peroxidasecoupled goat anti-rabbit secondary antibody (1:8000, ZSBio, Beijing, China) and EZ-ECL chemiluminescence Detection kit for HRP (Biological Industries, Beit-Haemek, Israel). The following primary antibodies were used: rabbit mAb PTEN (1:1000, Cell Signaling Technology, Danvers, MA, USA) and rabbit pAb $\beta$-actin (1:1000, Santa Cruz Biotechnology, Inc., Santa Cruz, CA, USA).

Cell proliferation assay. Aberrant cell proliferation is a hallmark of cancer. For proliferation assays, KLE cells were seeded in 96-well plates at 4,000 cells/well and, after $48 \mathrm{~h}$, were transfected with one of the following oligonucleotides: hsa-miR-21 mimic, hsa-miR-21 inhibitor and their respective SCs, using Lipofectamine 2000 Reagent. Cell proliferation was measured at 24,48 and $72 \mathrm{~h}$ after transfection, respectively, using the Cell Counting Kit-8 assay (CCK-8, Boster, Wuhan, China). The proliferation analysis of each group in each individual experiment and the whole experiment process were performed in triplicate. 
pGL3-PTEN-3'-UTR luciferase reporter assay. Using the computational algorithm RNA22 microRNA target detection (http://cbcsrv.watson.ibm.com/rna22.html), we characterized the putative binding sites in the 3'-UTR of PTEN mRNA. The fragment of the 3'-UTR of PTEN containing the putative miR-21 binding site was amplified from human genomic DNA by PCR, cloned into the $X b a 1$ site downstream of the luciferase reporter gene of the pGL3-Control vector (Promega, Madison, WI, USA) and named wt-PTEN-3'-UTR-pGL3. For the control, we introduced three point mutations into the seed region of the miR-21 binding sites and named the vector mu-PTEN-3'-UTRpGL3. KLE cells were seeded in 24 -well plates $\left(1 \times 10^{5}\right.$ cells/ well). After $48 \mathrm{~h}$, the cells were co-transfected with wt-PTEN3'-UTR-pGL3 or mu-PTEN-3'-UTR-pGL3 vectors, pRL-TK Renilla Luciferase Reporter Vector (Promega), hsa-miR-21 mimic, hsa-miR-21 inhibitor or their respective SC using Lipofectamine 2000 Reagent. Luciferase activities were measured using the Dual-Luciferase ${ }^{\circledR}$ Reporter Assay system (Promega) $48 \mathrm{~h}$ after transfection. The firefly luciferase activity was normalized to renilla luciferase actvity for each sample. All the experiments were performed in triplicate

Statistical analysis. Statistical analysis was performed using SPSS 13.0. Data are expressed as the mean \pm standard error of the mean (SEM) of three independent experiments. Pearson's product-moment correlation coefficient was used to assess the correlation between PTEN mRNA and protein levels and miR-21 levels in EEC. A paired Student's t-test was used to evaluate differences between two groups. Multiple group comparisons were analyzed using ANOVA with a post hoc test. $\mathrm{P}<0.05$ was considered to indicate a statistically significant result.

\section{Results}

Inverse correlation of miR-21 levels with PTEN protein expression in EEC. miR-21 was overexpressed in all 16 EEC tumor tissues compared with the matched adjacent non-tumor tissues (Fig. 1A, Table III). The average fold change of miR-21 was 2.985-fold (range, 1.021-7.402-fold; SEM, 0.419) higher in EEC tumor tissue versus non-tumor tissues. Compared with the non-tumor tissues, the EEC tumor tissue expressed a significantly lower level of PTEN protein (1.02 \pm 0.06 vs. $0.57 \pm 0.08, \mathrm{P}=0.022$, paired Student's t-test, Fig. 1B). Fig.1C reveals representative gels for PTEN protein levels in 8 pairs of tumor tissues and matched non-tumor tissues from EEC patients. A significantly lower level of PTEN mRNA was observed $\left(7.84 \pm 0.82 \times 10^{-3}\right.$ vs. $5.75 \pm 0.58 \times 10^{-3}, \mathrm{P}=0.047$, paired Student's t-test, Fig. 1D). Furthermore, a significant inverse correlation was observed between miR-21 and PTEN protein expression (Fig. 1E), but not between miR-21 and PTEN mRNA expression (Pearson's correlation $=0.453, \mathrm{P}=0.078$ ).

miR-21 mimic suppresses and miR-21 inhibitor enhances PTEN protein levels without affecting $m R N A$ levels in EEC. To investigate the regulation of miR-21 to PTEN, we designed experiments of gain- and loss-of-function of miR-21 in the KLE cell line. An miR-21 mimic, inhibitor or their respective SC were transfected into KLE cells and the efficiency of transfection was $\sim 90 \%$. As shown in Fig. 2A, the administration of miR-21 mimic resulted in a $6.898 \pm 0.312$-fold upregulation
Table III. miR-21 expression and clinicopathological factors. miR-21/U6 snRNA

Clinicopathological factors $\quad \mathrm{n} \quad\left(\right.$ mean \pm SEM; $\left.x 10^{-4}\right)$

$\begin{array}{lll}\text { Clinical stage } & & \\ \text { I } & 8 & 2.62 \pm 0.33 \\ \text { II } & 4 & 4.81 \pm 0.15 \\ \text { III } & 4 & 7.38 \pm 0.82\end{array}$

Histological grade

$\begin{array}{lll}\text { G1 } & 3 & 1.78 \pm 0.43 \\ \text { G2 } & 9 & 4.54 \pm 0.48 \\ \text { G3 } & 4 & 5.88 \pm 1.55\end{array}$

Lymph node metastasis

$\begin{array}{lrr}\text { Negative } & 14 & 3.80 \pm 1.06 \\ \text { Positive } & 2 & 8.28 \pm 0.46\end{array}$

Myometrial invasion

$<1 / 2$ myometrial thickness $\quad 10 \quad 3.45 \pm 0.58$

$>1 / 2$ myometrial thickness $6 \quad 5.86 \pm 0.90$

$(\mathrm{P}=0.003)$ and administration of the miR-21 inhibitor resulted in a $33.4 \pm 5.4 \%$ decrease $(\mathrm{P}=0.007)$ in $\mathrm{miR}-21$ expression. However, there was no significant difference between SCs and negative control $(\mathrm{P}=0.862$ for $\mathrm{miR}-21 \mathrm{mimic} \mathrm{SC}$ compared with negative control and $\mathrm{P}=0.953$ for $\mathrm{miR}-21$ inhibitor $\mathrm{SC}$ compared with negative control).

In western blot analysis, the upregulation of miR-21 in the miR-21 mimic-transfected group led to a significant decrease in PTEN protein expression level, to $53.8 \pm 1.6 \%$ of that of the control $(\mathrm{P}=0.007)$, and the downregulation of miR-21 in the miR-21 inhibitor-transfected group led to a significant increase in PTEN protein level, to $1.888 \pm 0.085$-fold that of the control $(\mathrm{P}=0.002$, Fig. 2B and $\mathrm{C})$; however, there was no significant difference between $\mathrm{SC}$ and negative control $\mathrm{P}=0.517$ for the miR-21 mimic SC compared with negative control and $\mathrm{P}=0.315$ for miR-21 inhibitor SC compared with negative control). In qRT-PCR analysis, no statistically significant change in PTEN mRNA expression was observed among those groups $(\mathrm{P}>0.05$, Fig. 2D). These results indicated that the expression of PTEN protein, but not mRNA, was regulated by miR-21 in KLE cells.

miR-21 directly targets the 3'-UTR of PTEN mRNA. To validate the putative direct binding site of miR-21 in the 3'-UTR of PTEN mRNA, a dual-luciferase reporter assay was carried out. The putative binding sites characterized by the computational algorithm RNA22 microRNA target detection are shown in Fig. 3A. The results of the luciferase assay revealed that, compared with negative control and SCs, the expression of luciferase in the wt-PTEN-3'-UTR-pGL3 group was downregulated to $62.93 \%$ of that of the control in the presence of the miR-21 mimic (both $\mathrm{P}=0.001$, Fig. 3B). The effect was reversed (luciferase expression $95.89 \%$ of that of the control) when three point mutations were introduced to the seed region of the miR-21 binding sites (Fig. 3B) and rescued (90.43\%) by administration of the miR-21 inhibitor (Fig. 3C). Taken together, these results confirm that PTEN is directly regulated by $\mathrm{miR}-21$ in the KLE cell line. 
A

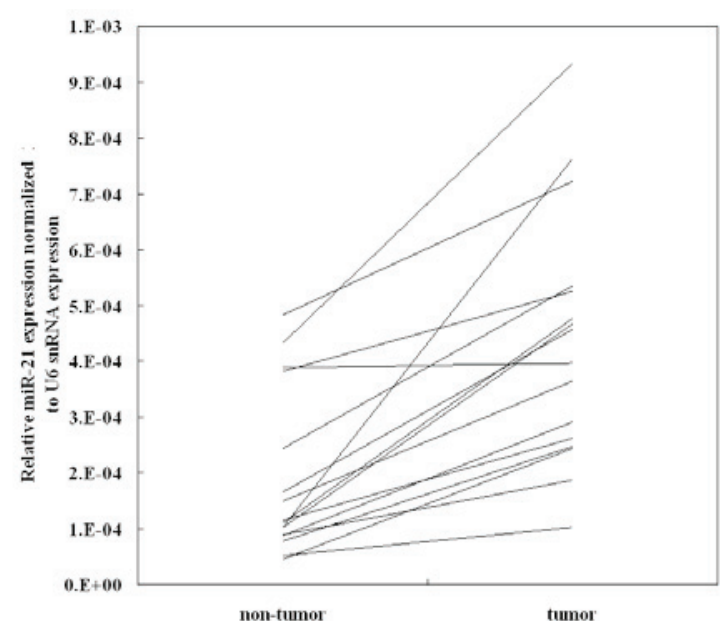

C

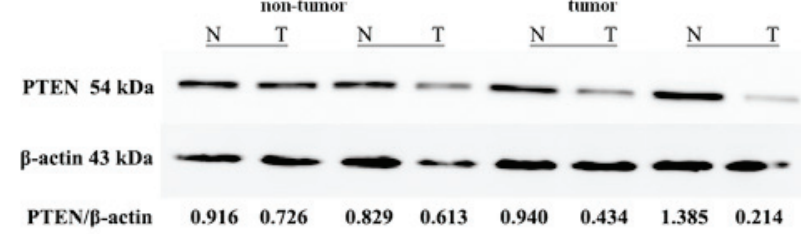

D

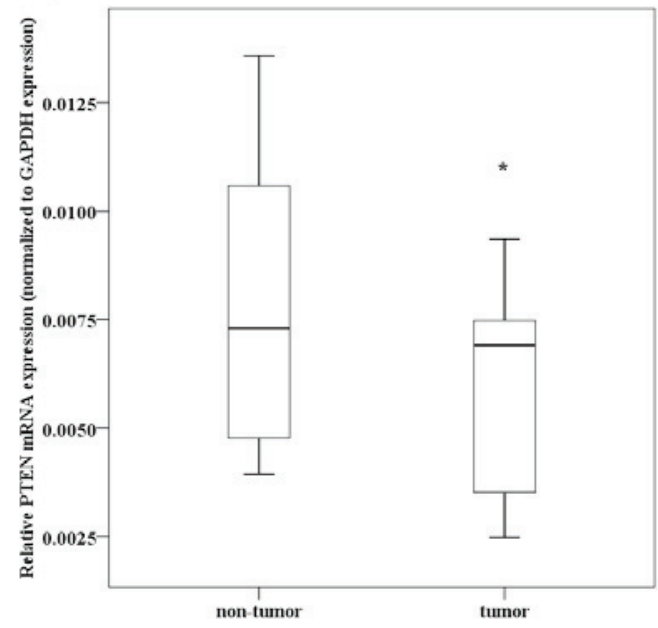

B

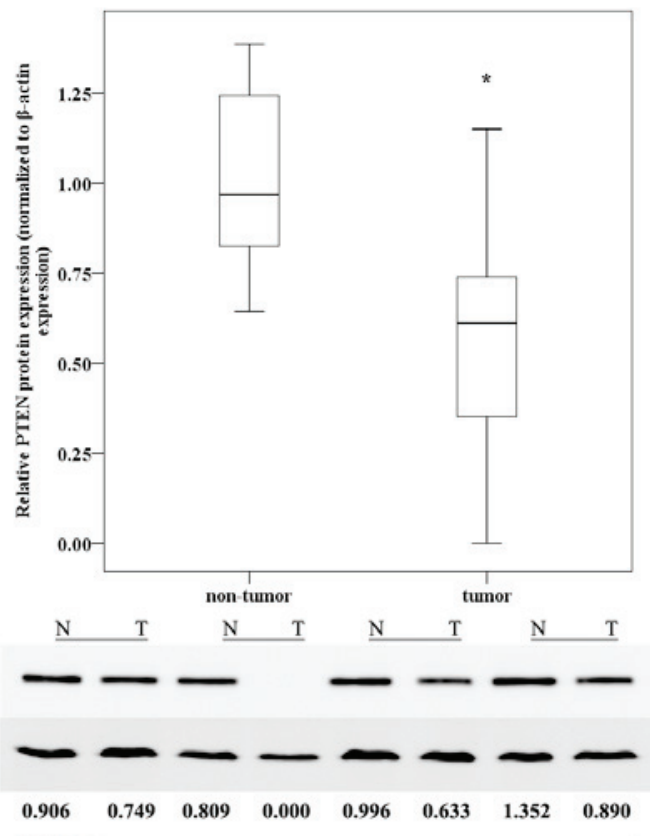

$\mathbf{E}$

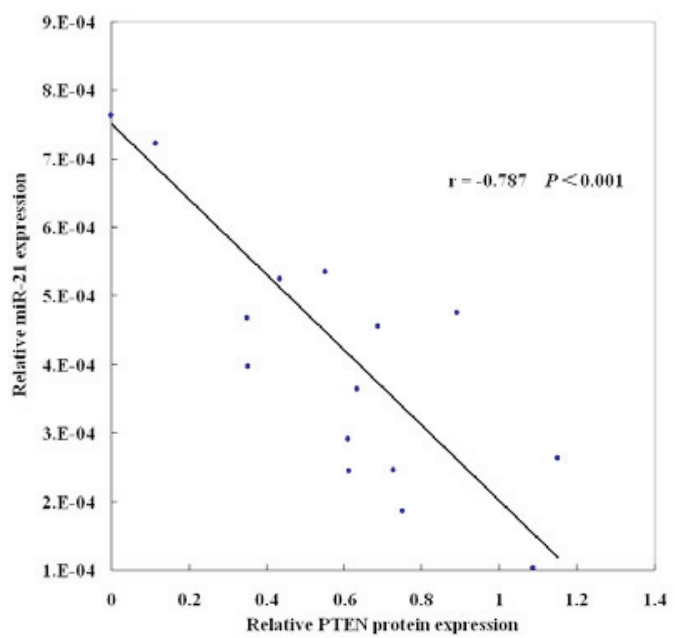

Figure 1. Expression of miR-21 and its inverse correlation with PTEN protein in EEC tissues. Sixteen pairs of matched EEC specimens were detected to determine the expression levels of PTEN mRNA and protein. (A) miR-21 was found to be overexpressed in EEC tumor tissues compared with paired non-tumor tissues detected by qRT-PCR. Relative miR-21 expression was normalized to U6 snRNA expression. (B) Relative expression of PTEN protein in EEC tumor tissues compared with matched non-tumor tissues $(\mathrm{n}=16, \mathrm{P}=0.022$, paired Student's t-test). PTEN protein expression was detected by western blot analysis and normalized to $\beta$-actin protein expression. (C) Representative gels for PTEN protein levels in 8 pairs of tumor tissues and matched non-tumor tissues. $\mathrm{N}$, non-tumor tissue; $\mathrm{T}$, tumor tissue. (D) Relative expression of PTEN mRNA in EEC tumor tissues compared with matched non-tumor tissues ( $\mathrm{n}=16$, $\mathrm{P}=0.047$, paired Student's t-test). The PTEN-mRNA levels were evaluated by qRT-PCR and normalized to GAPDH mRNA expression. (E) Correlation between relative miR-21 expression and PTEN protein levels in EEC tissues (Pearson's correlation $=-0.787, \mathrm{P}<0.001$ ). ${ }^{\mathrm{P}}<0.01$. miR, microRNA, EEC, endometrioid endometrial cancer; snRNA, small nuclear RNA.

Overexpression of miR-21 increases and inhibition of $m i R-21$ decreases cell proliferation. To evaluate the potential oncogenic effect of miR-21 on endometrial tumor cell proliferation, we performed an overexpression study, using an miR-21 mimic, and an inhibition study, with an miR-21 inhibitor. In the KLE cell line, the miR-21 mimic increased and miR-21 inhibitor decreased cell proliferation significantly (Fig. 4). These results indicate that the expression of miR-21 modulates endometrial tumor cell proliferation.

\section{Discussion}

The altered expression of miRNAs, including miR-21, has been found to be associated with various disorders, most specifically with cancer (6-15). The over- or underexpression of miRNAs is thought to result in down- or upregulation of the protein product of the target genes, thus affecting various biological behaviors of tumorigenesis (4). miRNA microarray analyses have shown that a series of miRNAs are highly expressed in EEC. For example, the miR-200 family has been reported to be highly expressed in EEC compared with normal endometrial tissues and may play an important role in cancer growth (17). In uterine disorders, miR-21 has been found to be associated with endometriosis, leiomyoma and cervical cancer $(18,19)$. However, the exact role of miR-21 in EEC is not fully understood.

In the present study, quantitative RT-PCR assay confirmed that miR-21 was overexpressed in EEC tissues compared with their adjacent matched non-tumor endometrium. This was 
A

C

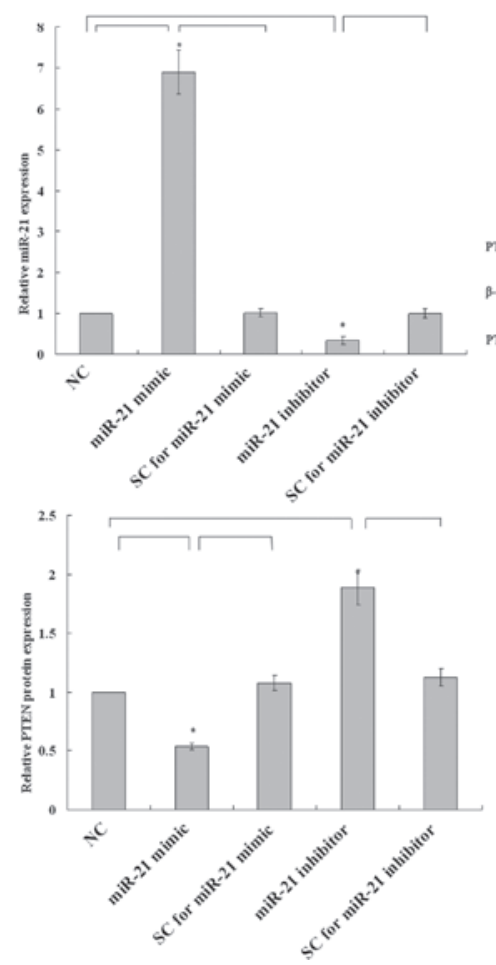

B

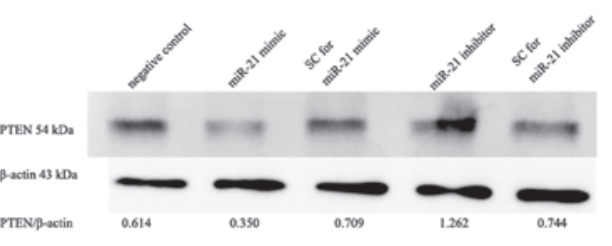

D

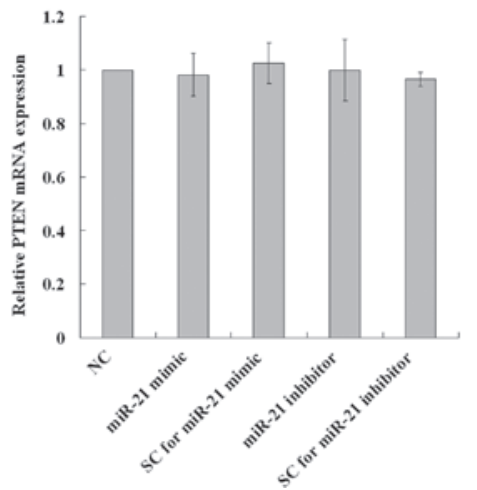

Figure 2. Detection of PTEN protein and mRNA in transfected KLE cells. (A) The level of miR-21 expression in KLE cells $48 \mathrm{~h}$ after transfection. (B) Western blotting gel for PTEN protein level in KLE $72 \mathrm{~h}$ after transfection. (C) The relative expression of PTEN protein normalized to $\beta$-actin. There is a statistically significant difference between groups transfected with miR-21 mimic, miR-21 inhibitor, their respective SC and NC. (D) Levels of PTEN mRNA in KLE cells detected by qRT-PCR at $48 \mathrm{~h}$ following transfection. Mean \pm SEM. $\mathrm{n}=3$. ${ }^{*} \mathrm{P}<0.01$. miRNA, microRNA; SC, scrambled control; NC, negative control.

A

Hsa-miR-21

3' AGUUGUAGUCAGACUAUUCGAU 5

III

111 IIIII

Position 1587 to 1608 of PTEN 3'-UTR 5'...UACAACUACUA-

$1 \downarrow \downarrow$

Mutant PTEN 3'-UTR:

5'... UACAACUACUA-UUGTAAUCGUA ... $3^{\prime}$

B

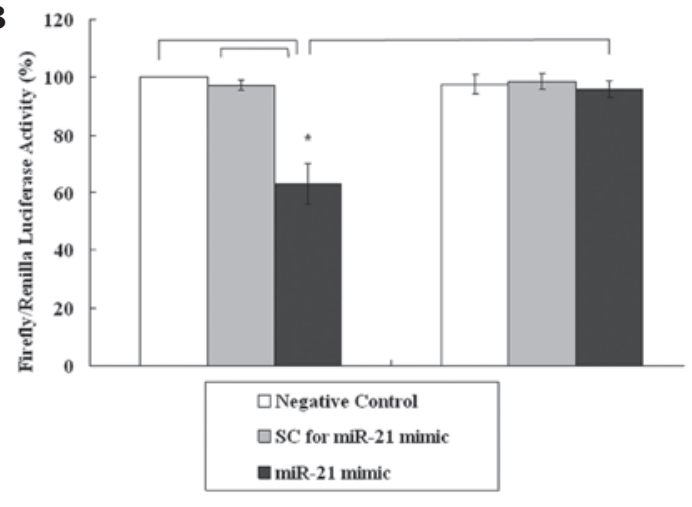

C

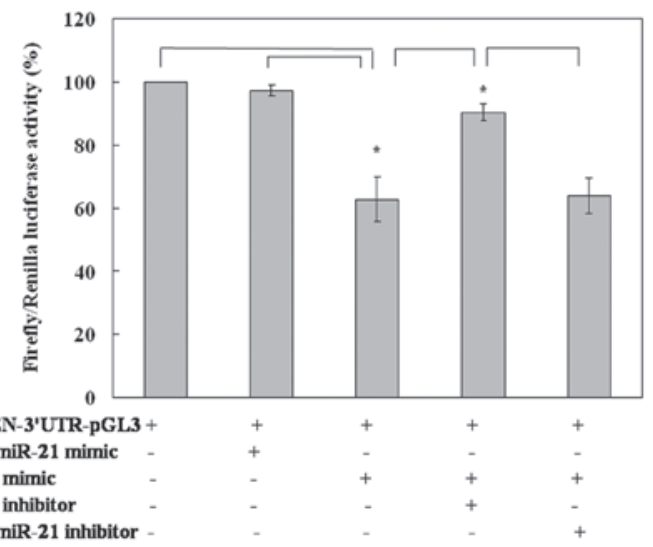

Figure 3. 3'-UTR of PTEN mRNA was targeted by miR-21. (A) Putative miR-21 binding sites on 3'-UTR of PTEN mRNA were predicted by the computational algorithm RNA22 microRNA target detection. The arrows show the mutant nucleotides. (B) Either the wild-type or mutant PTEN-3'-UTR-pGL3 was cotransfected into KLE cells with miR-21 mimic or SC for miR-21 mimic. The normalized luciferase activity in the negative control group was set as $100 \%$. Co-transfection of miR-21 mimic significantly decreased luciferase activity of wt-PTEN-3'-UTR-pGL3. However, this effect was reversed when mu-PTEN3'-UTR-pGL3 was co-transfected with the miR-21 mimic. (C) In the wt-PTEN-3'-UTR-pGL3-transfected KLE cells, miR-21 mimic or miR-21 inhibitor and their respective SC were co-transfected in KLE cells. miR-21 mimic significantly decreased the luciferase activity of wt-PTEN-3'-UTR-pGL3, while this effect was rescued by administration of the miR-21 inhibitor. Mean \pm SEM. n=3. 'P<0.01. 3'-UTR, 3' untranslated region; miR, microRNA; SC, scrambled control.

consistent with the results of the study by Torres et al (16). However, the results were not supported by previous miRNA profiling studies showing the dysregulated miRNAs in tumorous endometrium (20). This may be due to most of the studies not including paired self-controls and there may be individual differences in miRNA expressions. Our selfcontrol study overcame this disadvantage. Moreover, the qRT-PCR technique only detects mature miRNAs, but the microarray technique detects both precursor and mature miRNAs (21). We also revealed a trend that miR-21 expres- 


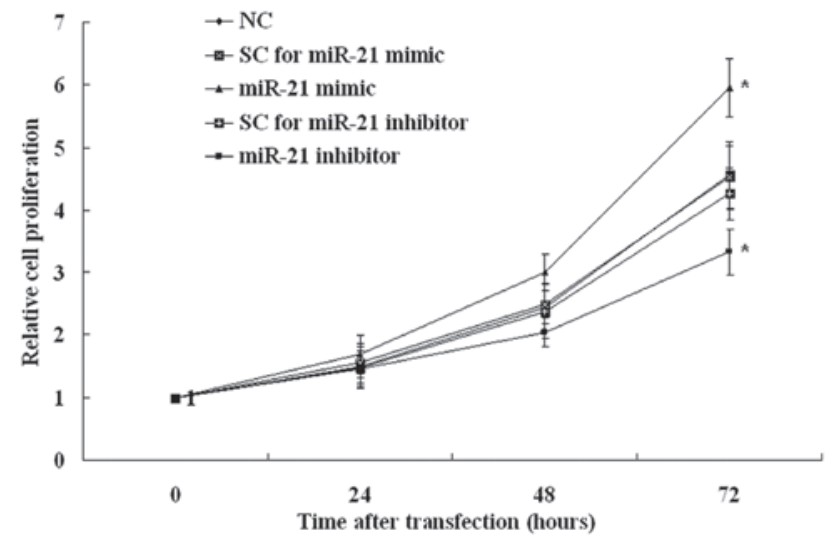

Figure 4. Cell proliferation modulated by miR-21 expression was evaluated by CCK- 8 assay 24,48 and $72 \mathrm{~h}$ after transfection in KLE cells. The results were expressed as fold change relative to the initial cell numbers. miR-21 mimic transfection significantly promoted tumor cell proliferation compared with the NC and SC, while the miR-21 inhibitor showed the opposite effect. All data are representative of three independent experiments. Values at $72 \mathrm{~h}$ were statistically significant. " $\mathrm{P}<0.01$. miR, microRNA; $\mathrm{NC}$, negative control; SC, scrambled control.

sion may be correlated with advanced clinical stages, deep myometrial invasion and high histological grade, which was not consistent with the results of the study by Torres et al (16). However, larger sample numbers and further studies are required to reveal the clinical relevance of these results. Furthermore, miR-21 has been shown to be overexpressed in a variety of solid tumors (22). Correlations between altered miR-21 expression and malignancy-related cellular processes, including proliferation, migration and apoptosis, have been well demonstrated $(6,10,19)$. These correlations indicate that the upregulation of miR-21 in EEC participates in the pathogenesis of endometrial cancer.

In the present study, we found that PTEN protein and mRNA expression were decreased in EEC tumor tissues compared with matched non-tumor tissues. Although several studies have revealed various frequencies of somatic PTEN mutation in EEC (2), the values are not adequate to explain PTEN inactivation in EEC and in several other tumor types (3). Other mechanisms participate in the loss of PTEN protein. PTEN has been validated as a target gene of miR-21 in a variety of malignancies $(5,6,9,23)$ but has not been reported in EEC. In the present study, matched analysis of miR-21 and PTEN protein expression in tumor tissues showed a significantly negative correlation. Thus, we postulated that PTEN was also an important target of miR-21 in EEC. Although the negative correlation between miR-21 and PTEN levels suggests a regulatory role for miR-21 in PTEN expression, a conclusion cannot be drawn until direct evidence showing that the reduction or overexpression of miR-21 alters PTEN expression is found. In this regard, synthetic miR-21 mimic or inhibitor was transfected into the KLE cell line to test the effect of overexpression and inhibition of miR-21 on PTEN expression in EEC tumor cells. Our results showed direct evidence that miR-21 regulates PTEN expression at the protein, but not the mRNA, level. To further confirm that miR-21 directly targets the 3'-UTR of PTEN mRNA, we constructed luciferase expression plasmids containing the putative binding sites or mutated seed region sequences in the 3'-UTR of PTEN mRNA for miR-21. Our results verified that the PTEN 3'-UTR had target binding sites for miR-21.

In the present study, transfection of miR-21 increased the cell proliferation of the KLE cell line and transfection of the miR-21 inhibitor inhibited it. Given that PTEN expression and cell proliferation are regulated by miR-21 and that PTEN has been shown to repress tumor cell proliferation by blocking the PI3K/AKT pathway (24), it may be inferred that the alteration of cell growth modulated by the expression of miR-21 may be partially mediated by the PTEN/PI3K/ AKT pathway. Other putative targets besides PTEN or other miR-21-mediated mechanisms may also participate in the alteration of cell proliferation of the KLE cell line. Further research should be performed to elucidate those underlining mechanisms.

Several aspects of the mechanism of miR-21 dysregulation have been shown. In gastric cancer cells, the suppression of nuclear factor $\kappa$-light-chain-enhancer of activated B cells $(\mathrm{NF}-\kappa \mathrm{B})$ activation has been reported to enhance miR-21 expression and it has previously been found that NF- $\kappa \mathrm{B}$ may be activated by estrogen in endometrial cancer (25). The regulation of miRNA is a complex network. Further research is needed on the definite mechanism leading to the dysregulation of miR-21.

Above all, our study demonstrated that miR-21 was overexpressed in EEC and determined that this miRNA downregulates PTEN expression via binding to the 3'-UTR of PTEN mRNA. In addition, we found that miR-21 promoted cell proliferation in EEC cells. The characterization of miR-21 as an oncogene provides evidence of its potential as a therapeutic target in EEC.

\section{Acknowledgements}

This study was supported by a grant from the Scientific Research Foundation for Shandong Provincial Scientific and Technological Projects (2009GG10002056) and a grant from the Shandong Natural Science Foundation (no. ZR2010HM005).

\section{References}

1. Jemal A, Siegel R, Ward E and Xu J: Cancer statistics, 2010. CA Cancer J Clin 60: 277-300, 2010.

2. Tashiro H, Blazes MS, Wu R, et al: Mutations in PTEN are frequent in endometrial carcinoma but rare in other common gynecological malignancies. Cancer Res 57: 3935-3940, 1997.

3. Perren A, Weng LP, Boag AH, et al: Immunohistochemical evidence of loss of PTEN expression in primary ductal adenocarcinomas of the breast. Am J Pathol 155: 1253-1260, 1999.

4. Bartel DP: MicroRNAs: genomics, biogenesis, mechanism, and function. Cell 116: 281-297, 2004.

5. Lee YS and Dutta A: MicroRNAs in cancer. Annu Rev Pathol 4: 199-227, 2009.

6. Meng F, Henson R, Lang M, et al: Involvement of human micro-RNA in growth and response to chemotherapy in human cholangiocarcinoma cell lines. Gastroenterology 130: 2113-2129, 2006.

7. Meng F, Henson R, Wehbe-Janek H, et al: MicroRNA-21 regulates expression of the PTEN tumor suppressor gene in human hepatocellular cancer. Gastroenterology 133: 647-658, 2007.

8. Asangani IA, Rasheed SA, Nikolova DA, et al: MicroRNA-21 (miR-21) post-transcriptionally downregulates tumor suppressor Pdcd4 and stimulates invasion, intravasation and metastasis in colorectal cancer. Oncogene 27: 2128-2136, 2008. 
9. Frankel LB, Christoffersen NR, Jacobsen A, et al: Programmed cell death 4 (PDCD4) is an important functional target of the microRNA miR-21 in breast cancer cells. J Biol Chem 283 1026-1033, 2008.

10. Zhu S, Wu H, Wu F, et al: MicroRNA-21 targets tumor suppressor genes in invasion and metastasis. Cell Res 18: 350-359, 2008.

11. Yao Q, Xu H, Zhang QQ, et al: MicroRNA-21 promotes cell proliferation and down-regulates the expression of programmed cell death 4 (PDCD4) in HeLa cervical carcinoma cells. Biochem Biophys Res Commun 388: 539-542, 2009.

12. Si ML, Zhu S, Wu H, et al: miR-21-mediated tumor growth. Oncogene 26: 2799-2803, 2007.

13. Zhu S, Si ML, Wu H and Mo YY: MicroRNA-21 targets the tumor suppressor gene tropomyosin 1 (TPM1). J Biol Chem 282 14328-14336, 2007.

14. Qin W, Zhao B, Shi Y, et al: BMPRII is a direct target of miR-21. Acta Biochim Biophys Sin (Shanghai) 41: 618-623, 2009.

15. Sayed D, Rane S, Lypowy J, et al: MicroRNA-21 targets Sprouty2 and promotes cellular outgrowths. Mol Biol Cell 19: 3272-3282, 2008.

16. Torres A, Torres K, Paszkowski T, et al: Highly increased maspin expression corresponds with up-regulation of miR-21 in endometrial cancer: a preliminary report. Int J Gynecol Cancer 21: 8-14, 2011

17. Snowdon J, Zhang X, Childs T, et al: The microRNA-200 family is upregulated in endometrial carcinoma. PLoS One 6: e22828, 2011.
18. Ramón LA, Braza-Boïls A, Gilabert-Estellés J, et al: microRNAs expression in endometriosis and their relation to angiogenic factors. Hum Reprod 26: 1082-1090, 2011.

19. Pan Q, Luo $X$ and Chegini N: microRNA 21: response to hormonal therapies and regulatory function in leiomyoma, transformed leiomyoma and leiomyosarcoma cells. Mol Hum Reprod 16: 215-227, 2010.

20. Cohn DE, Fabbri M, Valeri N, et al: Comprehensive miRNA profiling of surgically staged endometrial cancer. Am J Obstet Gynecol 202: 656 e1-8, 2010.

21. Lee EJ, Gusev Y, Jiang J, et al: Expression profiling identifies microRNA signature in pancreatic cancer. Int J Cancer 120: 1046-1054, 2007.

22. Volinia S, Calin GA, Liu CG, et al: A microRNA expression signature of human solid tumors defines cancer gene targets. Proc Natl Acad Sci USA 103: 2257-2261, 2006.

23. Ma WJ,Lv GD, Tuersun A, et al: Role of microRNA-21 and effect on PTEN in Kazakh's esophageal squamous cell carcinoma. Mol Biol Rep 38: 3253-3260, 2011

24. Vogt PK, Gymnopoulos M and Hart JR: PI 3-kinase and cancer: changing accents. Curr Opin Genet Dev 19: 12-17, 2009.

25. Seo KH, Lee HS, Jung B, et al: Estrogen enhances angiogenesis through a pathway involving platelet-activating factor-mediated nuclear factor-kappaB activation. Cancer Res 64: 6482-6488, 2004. 